CUBO A Mathematical Journal Vol.14, $N^{0} 01$, (81-91). March 2012

\title{
Special Recurrent Transformation in an NPR-Finsler Space
}

\author{
ANJALI Goswami \\ Department of Mathematics \\ Jagannath Gupta Institute of Engineering and Technology \\ Sitapura, Jaipur, India \\ email: dranjaligoswami@rediffmail.com
}

\begin{abstract}
In this paper, an infinitesimal transformation $\bar{x}^{i}=x^{i}+\epsilon v^{i}\left(x^{j}\right)$, where the vector $v^{i}$ is recurrent has been considered in an NPR- Finsler space. Such transformation is being called special recurrent transformation if the recurrence vector of the NPRFinsler space is Lie invariant. Besides different properties of such transformation, the conditions for such transformation to be curvature collineation and an affine motion have been obtained.
\end{abstract}

\section{RESUMEN}

En este artículo se considera una transformación infinitesimal $\bar{\chi}^{i}=\chi^{i}+\epsilon v^{i}\left(\chi^{j}\right)$, donde el vector $v^{i}$ es recurrente, en un espacio NPR- Finsler. Tal transformación se dice transformación recurrente especial si el vector recurrente del espacio NPR- Finsler es Lie invariante. Además se han obtenido diferentes propiedades de dicha transformación y las condiciones para que ésta sea una colineación de curvatura y una moción afín.

Keywords and Phrases: NPR-Finsler space, recurrent vector fields, special recurrent transformation, curvature collineation, affine motion.

2010 AMS Mathematics Subject Classification: 53B40. 


\section{Introduction}

Let an n-dimensional Finsler space $F_{n}$ be equipped with fundamental metric function $F\left(x^{k}, \dot{x}^{k}\right)$, metric tensor $g_{i j}$ and Berwald connection $G_{j k}^{i}$. Covariant derivative of any tensor with respect to Berwald connection is given by [6]

$$
\mathfrak{B}_{k} T_{j}^{i}=\partial_{k} T_{j}^{i}-\left(\dot{\partial}_{r} T_{j}^{i}\right) G_{k h}^{r} \dot{x}^{h}+T_{j}^{r} G_{k r}^{i}-T_{r}^{i} G_{j k}^{r}
$$

where $\partial_{k} \equiv \frac{\partial}{\partial x^{k}}$ and $\dot{\partial}_{r} \equiv \frac{\partial}{\partial \dot{x}^{r}}$.

The commutation formulae for the operators $\mathfrak{B}_{k}$ and $\dot{\partial}_{k}$ are given by

$$
\begin{gathered}
\dot{\partial}_{j} \mathfrak{B}_{k} T_{h}^{i}-\mathfrak{B}_{k} \dot{\partial}_{j} T_{h}^{i}=T_{h}^{r} G_{j k r}^{i}-T_{r}^{i} G_{j k h}^{r}, \\
\mathfrak{B}_{j} \mathfrak{B}_{k} T_{h}^{i}-\mathfrak{B}_{k} \mathfrak{B}_{j} T_{h}^{i}=T_{h}^{r} H_{j k r}^{i}-T_{r}^{i} H_{j k h}^{r}-\left(\dot{\partial}_{r} T_{h}^{i}\right) H_{j k}^{r},
\end{gathered}
$$

where

$$
\begin{gathered}
G_{j k h}^{i}=\dot{\partial}_{h} G_{j k}^{i}, \\
H_{j k h}^{i}=\partial_{j} G_{k h}^{i}+G_{h r j}^{i} G_{k}^{r}+G_{r j}^{i} G_{k h}^{r}-j / k
\end{gathered}
$$

and

$$
H_{j k}^{i}=H_{j k h}^{i} \dot{x}^{h} \text {. }
$$

The symbol $-\mathrm{j} / \mathrm{k}$ means the subtraction of the earlier terms after interchanging $\mathrm{j}$ and $\mathrm{k}$. The tensor $\mathrm{G}_{\mathrm{j} k \mathrm{~h}}^{\mathrm{i}}$ is symmetric in its lower indices and satisfies

$$
G_{j k h}^{i} \dot{x}^{h}=G_{j h k}^{i} \dot{x}^{h}=G_{h j k}^{i} \dot{x}^{h}=0
$$

while the Berwald curvature tensor $\mathrm{H}_{j k h}^{i}$ satisfies

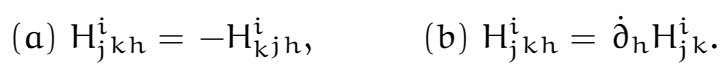

The Berwald deviation tensor $\mathrm{H}_{\mathrm{j}}^{\mathrm{i}}$ is defined by

$$
\text { (a) } H_{j}^{i}=H_{j k}^{i} \dot{x}^{k}, \quad \text { (b) } H_{j k}^{i}=1 / 3 \dot{\partial}_{k} H_{j}^{i}-j / k \text {. }
$$

Pandey[2] proved that the relation between the normal projective curvature tensor $\mathrm{N}_{\mathbf{j} k \boldsymbol{h}}^{i}$ defined by Yano [7] and the Berwald curvature tensor $H_{j k h}^{i}$ is given by

$$
\begin{gathered}
N_{j k h}^{i}=H_{j k h}^{i}-\frac{\dot{x}^{i}}{n+1} \dot{\partial}_{h} H_{j k r}^{r}, \\
N_{j k r}^{r}=H_{j k r}^{r} .
\end{gathered}
$$

The relation between the tensors $\mathrm{N}_{j k h}^{i}$ and $H_{j k}^{i}$ is given by

$$
N_{j k h}^{i} \dot{x}^{h}=H_{j k}^{i} \text {. }
$$




\section{An NPR-Finsler Space}

An NPR-Finsler space was defined by P. N. Pandey [2] in 1980. It is a Finsler space whose normal projective curvature tensor $\mathrm{N}_{j k h}^{i}$ satisfies

$$
\mathfrak{B}_{m} N_{j k h}^{i}=\lambda_{m} N_{j k h}^{i},
$$

where $\lambda_{m}$ is a covariant vector called recurrence vector. This vector is atmost a point function, i.e. independent of the directional arguments.

It was observed by P. N. Pandey [2] that the tensors $H_{j k}^{i}$ and $H_{j}^{i}$ are recurrent in NPR-Finsler space. Thus in an NPR-Finsler space, we have

$$
\text { (a) } \mathfrak{B}_{m} H_{j k}^{i}=\lambda_{m} H_{j k}^{i}, \quad \text { (b) } \mathfrak{B}_{m} H_{j}^{i}=\lambda_{m} H_{j}^{i} \text {. }
$$

However, an NPR-Finsler space is not necessarily a recurrent Finsler space. Also, a recurrent Finsler sapce is not necessarily an NPR-Finsler space. In another paper, P.N. Pandey [4] established the following identities:

$$
\begin{gathered}
\lambda_{m} N_{j k h}^{i}+\lambda_{j} N_{k m h}^{i}+\lambda_{k} N_{m j h}^{i}=0, \\
\lambda_{m} H_{j k h}^{i}+\lambda_{j} H_{k m h}^{i}+\lambda_{k} H_{m j h}^{i}=0, \\
\lambda_{m} H_{j k}^{i}+\lambda_{j} H_{k m}^{i}+\lambda_{k} H_{m j}^{i}=0,
\end{gathered}
$$

He further proved that in such space, the second Bianchi identity splits into the following identities:

$$
\begin{gathered}
\mathfrak{B}_{m} H_{j k h}^{i}+\mathfrak{B}_{j} H_{k m h}^{i}+\mathfrak{B}_{k} H_{m j h}^{i}=0, \\
H_{j k}^{r} G_{m h r}^{i}+H_{k m}^{r} G_{j h r}^{i}+H_{m j}^{r} G_{k h r}^{i}=0 .
\end{gathered}
$$

Contracting the indices in $(2.2 \mathrm{~b})$ and using $\mathrm{H}_{i}^{i}=(n-1) \mathrm{H}$, we get

$$
\mathfrak{B}_{\mathrm{m}} \mathrm{H}=\lambda_{\mathrm{m}} \mathrm{H} \text {. }
$$

Differentiating (2.8) covariantly with respect to $x^{h}$ and taking skew-symmetric part, we have

$$
\left(\mathfrak{B}_{h} \mathfrak{B}_{m}-\mathfrak{B}_{m} \mathfrak{B}_{h}\right) \mathrm{H}=A_{h \mathfrak{m}} \mathrm{H}
$$

where $A_{h \mathrm{~m}}=\mathfrak{B}_{\mathrm{h}} \lambda_{\mathrm{m}}-\mathfrak{B}_{\mathrm{m}} \lambda_{\mathrm{h}}$.

Using (1.3) in (2.9), we have

$$
-\dot{\partial}_{\mathrm{r}} \mathrm{H} \mathrm{H}_{\mathrm{hm}}^{\mathrm{r}}=\mathrm{A}_{\mathrm{hm}} \mathrm{H},
$$

which after further covariant differentiation gives

$$
-\left(\mathfrak{B}_{k} \dot{\partial}_{r} H\right) H_{h}^{r}=\left(\mathfrak{B}_{k} A_{h m}\right) H .
$$

Using the commutation formula (1.2) and the equation (2.10), we get

$$
\mathfrak{B}_{k} A_{h m}=\lambda_{k} A_{h m}
$$


provided $\mathrm{H}$ is non-vanishing. If we multiply (2.10) with $\lambda_{k}$ and take skew-symmetric part, we find

$$
\lambda_{k} A_{h m}+\lambda_{h} A_{m k}+\lambda_{m} A_{k h}=0
$$

provided $\mathrm{H} \neq 0$. Thus, we find that the recurrence vector $\lambda_{\mathrm{m}}$ of an NPR-Finsler space satisfies (2.12) and (2.13) provided $\mathrm{H} \neq 0$.

In view of the commutation formula given by (1.2), we get

$\dot{\partial}_{j} \mathfrak{B}_{m} \lambda_{k}-\mathfrak{B}_{m} \dot{\partial}_{j} \lambda_{k}=-\lambda_{r} G_{j m k}^{r}$

which due to the fact that the recurrence vector is independent of $\dot{\chi}^{i}$, gives

$$
\dot{\partial}_{j} \mathfrak{B}_{m} \lambda_{k}=-\lambda_{r} G_{j m k}^{r} .
$$

Taking skew-symmetric part of (2.14), we get

$$
\dot{\partial}_{j} A_{m k}=0 .
$$

Now

$$
\dot{\partial}_{j} \mathfrak{B}_{k} A_{h m}-\mathfrak{B}_{k} \dot{\partial}_{j} A_{h m}=-A_{r m} G_{j k h l}^{r}-A_{h r} G_{j k m}^{r}
$$

which, in view of (2.12) and (2.15), gives

$$
A_{r m} G_{j k h}^{r}+A_{h r} G_{j k m}^{r}=0 .
$$

\section{A Recurrent Vector Field in An NPR-Finsler space}

A vector field $v^{i}$ is called recurrent if it satisfies

$$
\mathfrak{B}_{k} v^{i}=\mu_{k} v^{i} .
$$

Differentiating (3.1) covariantly with respect to $x^{j}$ and using the commutation formula (1.3), we get

$$
H_{j k h}^{i} v^{h}=\mu_{j k} v^{i}
$$

where $\mu_{j k}=\mathfrak{B}_{j} \mu_{k}-\mathfrak{B}_{k} \mu_{j}$. The tensor $\mu_{j k}$ may or may not vanish. Let us consider the case when $\mu_{j k} \neq 0$. From (1.10) and (3.2), we find

$$
\left(N_{j k h}^{i}+\frac{\dot{x}^{i}}{n+1} \dot{\partial}_{h} N_{j k r}^{r}\right) v^{h}=\mu_{j k} v^{i} .
$$

Differentiating (3.3) covariantly with respect to $x^{\mathrm{m}}$, and using (2.1) and (3.1), we have

$$
\left(\lambda_{m} N_{j k h}^{i}+\frac{\dot{x}^{i}}{n+1} B_{m} \dot{\partial}_{h} N_{j k r}^{r}\right) v^{h}=v^{i} \mathfrak{B}_{m} \mu_{j k},
$$

which in view of (1.2), gives

$$
\lambda_{m}\left(N_{j k h}^{i}+\frac{\dot{x}^{i}}{n+1} \dot{\partial}_{h} N_{j k r}^{r}\right) v^{h}+\frac{\dot{x}^{i}}{n+1}\left(N_{s k r}^{r} G_{h m j}^{s}+N_{j s r}^{r} G_{h m k}^{s}\right) v^{h}=v^{i} \mathfrak{B}_{m} \mu_{j k} .
$$


From (3.3) and (3.5), we get

$$
\left(\lambda_{m} \mu_{j k}-\mathfrak{B}_{m} \mu_{j k}\right) v^{i}+\frac{\dot{x}^{i}}{n+1} v^{h}\left(N_{s k r}^{r} G_{h m j}^{s}+N_{j s r}^{r} G_{h m k}^{s}\right)=0 .
$$

Transvecting (3.6) by $y_{i}$ and using $y_{i} \dot{x}^{i}=F^{2}$, we get $\left(\lambda_{m} \mu_{j k}-\mathfrak{B}_{m} \mu_{j k}\right) y_{i} v^{i}+\frac{F^{2}}{n+1} v^{h}\left(N_{s k r}^{r} G_{h m j}^{s}+N_{j s r}^{r} G_{h m k}^{s}\right)=0$

which implies

$$
\frac{v^{h}}{n+1}\left(N_{s k r}^{r} G_{h m j}^{s}+N_{j s r}^{r} G_{h m k}^{s}\right)=\frac{1}{F^{2}}\left(\mathfrak{B}_{m} \mu_{j k}-\lambda_{m} \mu_{j k}\right) y_{i} v^{i} .
$$

Using (3.7) in (3.6), we get

$$
\left(\lambda_{m} \mu_{j k}-\mathfrak{B}_{m} \mu_{j k}\right) v^{i}-l^{i} l_{r} v^{r}\left(\lambda_{m} \mu_{j k}-\mathfrak{B}_{m} \mu_{j k}\right)=0
$$

where $l^{i}=\dot{x}^{i} / F$ and $l_{r}=y_{r} / F$.

(3.8) may be rewritten as

$\left(\lambda_{m} \mu_{j k}-\mathfrak{B}_{m} \mu_{j k}\right)\left(v^{i}-l^{i} l_{r} v^{r}\right)=0$.

This implies at least one of the conditions

$$
\text { (a) } \mathfrak{B}_{\mathrm{m}} \mu_{j k}=\lambda_{\mathrm{m}} \mu_{j k}, \quad \text { (b) } v^{i}=l^{i} l_{\mathrm{r}} v^{\mathrm{r}} \text {. }
$$

Suppose that the condition $\left(3.9\right.$ b)holds. Then the partial differentiation with respect to $\dot{x}^{h}$ gives

$$
0=\left(\dot{\partial}_{h} l^{i}\right) l_{r} v^{r}+l^{i}\left(\dot{\partial}_{h} l_{r}\right) v^{r}
$$

Using $\dot{\partial}_{h} l^{i}=\frac{1}{F}\left(\delta_{h}^{i}-l^{i} l_{h}\right)$ and $\dot{\partial}_{h} l_{r}=\frac{1}{F}\left(g_{h r}-l_{h} l_{r}\right)$ in (3.10),we find $0=\left(\delta_{h}^{i}-l^{i} l_{h}\right) l_{r} v^{r}+l^{i}\left(g_{h r}-l_{h} l_{r}\right) v^{r}$.

Contracting the indices $i$ and $h$ and using $\delta_{i}^{i}=n$ and $l^{r} l_{r}=1$, we get $(n-1) l_{r} v^{r}=0$.

This implies $l_{r} v^{r}=0$ for $n \neq 1$. In view of $l_{r} v^{r}=0,(3.9 b)$ gives $v^{i}=0$, a contradiction. Therefore $(3.9 \mathrm{~b})$ can not be true. Hence, we have (3.9a). From (2.4) and (3.2), we may deduce

$$
\lambda_{m} \mu_{j k}+\lambda_{j} \mu_{k m}+\lambda_{k} \mu_{m j}=0 .
$$

This leads to:

Theorem 3.1. In an NPR-Finsler space admitting a recurrent vector field $v^{i}$ given by (3.1), the tensor $\mu_{\mathrm{jk}}$ either vanishes identically or is recurrent and satisfies the identity (3.11).

Differentiating (3.1) partially with respect to $\dot{x}^{j}$ and using the commutation formula (1.2), we get

$$
\mathrm{G}_{j k r}^{i} v^{r}=\left(\dot{\partial}_{j} \mu_{k}\right) v^{i}
$$

Transvecting (2.17) by $v^{\mathrm{j}} \dot{\chi}^{\mathrm{m}}$ and using (3.12), we get

$$
A_{r m} v^{r} \dot{x}^{m} \dot{\partial}_{k} \mu_{h}=0 .
$$


This gives at least one of the following conditions:

$$
\text { (a) } A_{r m} v^{r} \dot{x}^{m}=0, \quad \text { (b) } \dot{\partial}_{k} \mu_{h}=0 \text {. }
$$

If (3.14a) holds, then its partial drivatives with respect to $\dot{x}^{k}$ gives

$$
A_{\mathrm{rk}} v^{r}=0
$$

Transvecting (2.13) by $v^{\mathrm{k}}$ and using (3.15), we find

$$
\lambda_{k} v^{k} A_{h m}=0 .
$$

Since $A_{h m} \neq 0$, we have

$$
\lambda_{k} v^{k}=0 .
$$

Thus we have

Theorem 3.2. In an NPR-Fnsler space admitting a recurrent vector field $v^{i}$ characterized by (3.1), we have at least one of the conditions (3.14b) and (3.17).

Suppose (3.14b) holds, then we have

$$
\dot{\partial}_{j} \mathfrak{B}_{k} \mu_{m}=-\mu_{r} G_{j k m}^{r} .
$$

Taking skew-symmetric part of (3.18) with respect to the indices $k$ and $m$, we get

$$
\dot{\partial}_{j} \mu_{k m}=0 \text {. }
$$

Differentiating (3.19) covariantly with respect to $x^{\mathrm{h}}$ and using commutation formula exhibitted by (1.2) and the equation (3.9a), we find $\mu_{r m} G_{k j h}^{r}+\mu_{k r} G_{m j h}^{r}=0$.

\section{A Special Recurrent Transformation}

An infinitesimal transformation

$$
\bar{x}^{i}=x^{i}+\epsilon v^{i}\left(x^{j}\right)
$$

where $v^{i}$ is a covariant vector field and $\epsilon$ is an infinitesimal constant, is called a special recurrent transformation if the vector field $v^{i}$ is recurrent and the transformation does not deform the recurrence vector $\lambda_{m}$ of the NPR-Finsler space, i.e. if the vector field $v^{i}$ satisfies (3.1) and

$$
£ \lambda_{\mathrm{m}}=0
$$

where $£$ is the operator of Lie differentiation with respect to the infinitesimal transformation (4.1). The necessary and sufficient condition for (4.1) to be an affine motion is given by

$$
£ \mathrm{G}_{j k}^{i}=0 .
$$


Since every affine motion is a curvature collination, (4.3) implies

$$
£ \mathrm{H}_{\mathrm{jkh}}^{\mathrm{i}}=0 .
$$

Operating (1.10) by the operator $£$ and using (4.4), we get

$$
£ \mathrm{~N}_{j k h}^{i}=-\frac{\dot{x}^{i}}{n+1} £ \dot{\partial}_{h} H_{j k r}^{r}
$$

Since the operators $£$ and $\dot{\partial}_{h}$ are commutative, (4.5) becomes $£ N_{j k h}^{i}=-\frac{\dot{x}^{i}}{n+1} \dot{\partial}_{h} £ H_{j k r}^{r}$ which in view of (4.4), gives

$$
£ \mathrm{~N}_{\mathrm{jkh}}^{i}=0 .
$$

Let us consider an NPR-Finsler space admitting an affine motion. Then we have (2.1), (4.3), (4.4) and (4.6).

Operating (2.1) by the operator $£$ and using (4.6), we have

$$
£ \mathfrak{B}_{m} N_{j k h}^{i}=\left(£ \lambda_{m}\right) N_{j k h}^{i} .
$$

In view of the commutation formula

$$
£ \mathfrak{B}_{k} T_{j}^{i}-\mathfrak{B}_{k} £ T_{j}^{i}=T_{j}^{r} £ G_{r k}^{i}-T_{r}^{i} £ G_{j k}^{r}-\left(\dot{\partial}_{r} T_{j}^{i}\right) £ G_{k s}^{r} \dot{x}^{s}
$$

and equations (4.3) and (4.6), the equation (4.7) gives (4.2) for $\mathrm{N}_{\mathrm{jkh}}^{i} \neq 0$. Thus, we obsereve that every affine motion generated by a recurrent vector field in an NPR-Finsler space is a special recurrent transformation. Now, we wish to discuss its converse problem.

Let us consider a special recurrent transformation (4.1) in an NPR-Finsler space. This transformation is characterized by (3.1) and (4.2). In view of theorem (3.2), we have at least one of the equations (3.14b) and (3.17). If (3.14b) does not hold, we must have (3.17), i.e. $\mathrm{L}=\lambda_{\mathrm{r}} v^{r}=0$. We shall divide the special recurrent transformations in two classes according as $\mathrm{L} \neq 0$ and $\mathrm{L}=0$. A special recurrent transformation is called of first kind if $L \neq 0$ while it is called of second kind if $\mathrm{L}=0$.

Let us consider a special recurrent transformation of the first kind. For such transformation $\mathrm{L} \neq 0$. Therefore in view of Theorem (3.2), the vector field $\mu_{k}$ must be a point function, i.e. $\dot{\partial}_{j} \mu_{k}=0$. Expanding the left hand side of equation (4.2) with the help of the formula

$$
£ T_{j}^{i}=v^{r} \mathfrak{B}_{r} T_{j}^{i}-T_{j}^{r} \mathfrak{B}_{r} v^{i}+T_{r}^{i} \mathfrak{B}_{j} v^{r}+\left(\dot{\partial}_{r} T_{j}^{i}\right) \mathfrak{B}_{s} v^{r} \dot{\chi}^{s}
$$

we get

$$
\nu^{r} \mathfrak{B}_{r} \lambda_{m}+L \mu_{m}=0
$$

Also

$$
\mathfrak{B}_{\mathrm{m}} \mathrm{L}=\mathfrak{B}_{\mathrm{m}}\left(\lambda_{\mathrm{r}} \nu^{\mathrm{r}}\right)=\nu^{\mathrm{r}} \mathfrak{B}_{\mathrm{m}} \lambda_{\mathrm{r}}+\mathrm{L} \mu_{\mathrm{m}} .
$$

Using (4.10) in (4.11), we have

$$
v^{r} A_{r k}+\mathfrak{B}_{m} L=0 .
$$


Differentiating (2.3) covariantly with respect to $x^{p}$ and using (2.1), we have

$$
\left(\mathfrak{B}_{p} \lambda_{m}\right) N_{j k h}^{i}+\left(\mathfrak{B}_{p} \lambda_{j}\right) N_{k m h}^{i}+\left(\mathfrak{B}_{p} \lambda_{k}\right) N_{m j h}^{i}=0
$$

Transvecting (4.13) by $v^{p}$ and using (4.10), we get

$$
\mu_{m} N_{j k h}^{i}+\mu_{j} N_{k m h}^{i}+\mu_{k} N_{m j h}^{i}=0
$$

Differentiating (2.11) and (2.13) covariantly with respect to $\chi^{\mathrm{p}}$ and then multiplying by $v^{\mathrm{p}}$, we get $\left(v^{p} \mathfrak{B}_{p} \lambda_{k}\right) A_{h m}+\left(v^{p} \mathfrak{B}_{p} \lambda_{h}\right) A_{m k}+\left(v^{p} \mathfrak{B}_{p} \lambda_{m}\right) A_{k h}=0$,

and $\left(v^{p} \mathfrak{B}_{p} \lambda_{k}\right) \mu_{h m}+\left(v^{p} \mathfrak{B}_{p} \lambda_{h}\right) \mu_{m k}+\left(v^{p} \mathfrak{B}_{p} \lambda_{m}\right) \mu_{k h}=0$, which imply

$$
\mu_{k} A_{h m}+\mu_{h} A_{m k}+\mu_{m} A_{k h}=0
$$

and

$$
\mu_{k} \mu_{h m}+\mu_{h} \mu_{m k}+\mu_{m} \mu_{k h}=0
$$

since $\mathrm{L} \neq 0$.

This proves the following:

Theorem 4.1. An NPR-Finsler space admitting a special recurrent transformation admits the identities (4.14), (4.15) and (4.16) provided $\mathrm{L} \neq 0$.

The commutation formula for the operators $£$ and $\mathfrak{B}_{k}$ in case of the recurrence vector $\lambda_{m}$ is given by

$£ \mathfrak{B}_{\mathrm{k}} \lambda_{\mathrm{m}}-\mathfrak{B}_{\mathrm{k}} £ \lambda_{\mathrm{m}}=-\lambda_{\mathrm{r}} £ \mathrm{G}_{\mathrm{mk}}^{\mathrm{r}}$

which, in view of (4.2), gives

$$
£ \mathfrak{B}_{\mathrm{k}} \lambda_{\mathrm{m}}=-\lambda_{\mathrm{r}} £ \mathrm{G}_{\mathrm{mk}}^{\mathrm{r}} \text {. }
$$

Taking skew-symmetric part of (4.17), we get

$$
£ A_{m k}=0 \text {. }
$$

Transvecting (4.14) by $\dot{x}^{h}$ and using (1.12), we get

$$
\mu_{m} H_{j k}^{i}+\mu_{j} H_{k m}^{i}+\mu_{k} H_{m j}^{i}=0 .
$$

Now $£ H_{j k}^{i}=L H_{j k}^{i}+\mu H_{j k r}^{i} v^{r}-\mu_{r} H_{j k}^{r} v^{i}+\mu_{j} H_{r k}^{i} v^{r}+\mu_{k} H_{j r}^{i} v^{r}$.

Transvecting (4.19) by $v^{\mathrm{m}}$ and using (3.2) in the above equation, we get $£ H_{j k}^{i}=\left(L+\mu_{m} v^{m}\right) H_{j k}^{i}+\left(\mu \mu_{j k}-\mu_{r} H_{j k}^{r}\right) v^{i}$.

This shows that $£ \mathrm{H}_{j k}^{i}=0$ if

$$
\mathrm{L}+\mu_{\mathrm{m}} v^{\mathrm{m}}=0 \text { and } \quad \mu \mu_{j k}-\mu_{\mathrm{r}} \mathrm{H}_{j k}^{\mathrm{r}}=0 \text {. }
$$

We know that $£ \mathrm{H}_{j k}^{i}=0$ is equivalent to $£ \mathrm{H}_{j k h}^{i}=0$.

Therefore we have: 
Theorem 4.2. A special recurrent transformation of the first kind is a curvature collineation if (4.20) holds.

The Lie derivative of $G_{j k}^{i}$ is given by

$$
£ G_{j k}^{i}=\mathfrak{B}_{j} \mathfrak{B}_{k} v^{i}+H_{m j k}^{i} v^{m}+G_{j k r}^{i} \mathfrak{B}_{s} v^{r} \dot{\chi}^{s},
$$

which in the present case is given by

$$
£ G_{j k}^{i}=\left(\mathfrak{B}_{j} \mu_{k}+\mu_{j} \mu_{k}\right) v^{i}+H_{m j k}^{i} \nu^{m},
$$

for $G_{j k r}^{i} v^{r}=\dot{\partial}_{j} \mu_{k} v^{i}=0$.

Differentiating (2.4) covariantly with respect to $x^{\mathfrak{p}}$ and transvecting by $\nu^{\mathfrak{p}}$, we ge $\left(\nu^{\mathfrak{p}} \mathfrak{B}_{\mathfrak{p}} \lambda_{\mathfrak{m}}\right) H_{j k h}^{i}+\left(\nu^{\mathfrak{p}} \mathfrak{B}_{p} \lambda_{j}\right) H_{k m h}^{i}+\left(\nu^{\mathfrak{p}} \mathfrak{B}_{p} \lambda_{k}\right) H_{m j h}^{i}=0$.

Using (4.10) in it, we find

$$
\mu_{m} H_{j k h}^{i}+\mu_{j} H_{k m}^{i}+\mu_{k} H_{m j}^{i}=0
$$

for $\mathrm{L} \neq 0$.

Transvecting (2.4) and (4.23) by $v^{\mathrm{m}}$ and adding, we get $\left(\lambda_{k}+\mu_{k}\right) H_{m j h}^{i} v^{m}-\left(\lambda_{j}+\mu_{j}\right) H_{m k h}^{i} v^{m}=0$.

From this we may conclude

$$
H_{m j h}^{i} v^{m}=\phi\left(\lambda_{j}+\mu_{j}\right) X_{h}^{i} .
$$

for some tensor $X_{h}^{i}$. Therefore

$$
£ G_{j k}^{i}=\left(\mathfrak{B}_{j} \mu_{k}+\mu_{j} \mu_{k}\right) v^{i}+\phi\left(\lambda_{j}+\mu_{j}\right) X_{k}^{i} .
$$

From this we find that the special recurrent transformation is affine motion if $\left(\mathfrak{B}_{j} \mu_{k}+\mu_{j} \mu_{k}\right) v^{i}=-\phi\left(\lambda_{j}+\mu_{j}\right) X_{k}^{i}$.

Now we consider a special recurrent transformation of the second kind $(\mathrm{L}=0)$. Transvecting $(2.5)$ by $v^{m}$ and using $L=\lambda_{m} v^{m}=0$, we get

$$
\lambda_{j} H_{k m}^{i} v^{m}+\lambda_{k} H_{m j}^{i} v^{m}=0 .
$$

This is possible only when

$$
H_{m k}^{i} v^{m}=\lambda_{k} X^{i}
$$

for some vector field $X^{i}$. Since $y_{i} H_{j k}^{i}=0, y_{i} X^{i}=0$.

$£ \mathrm{H}_{\mathrm{jk}}^{\mathrm{i}}$, in view of $(2.2),(3.1)$ and $(3.17)$, becomes

$$
£ H_{j k}^{i}=\mu H_{j k r}^{i} \nu^{r}-H_{j k}^{r} \mu_{r} \nu^{i}+\mu_{j} H_{r k}^{i} \nu^{r}+\mu_{k} H_{j r}^{i} \nu^{r}
$$

where $\mu=\mu_{k} \dot{x}^{k}$.

Using (3.2) and (4.9) in (4.10), we get

$$
£ H_{j k}^{i}=\left(\mu \mu_{j k}-\mu_{r} H_{j k}^{r}\right) v^{i}+\left(\mu_{j} \lambda_{k}-\mu_{k} \lambda_{j}\right) X^{i} .
$$


This shows that $£ \mathrm{H}_{j k}^{i}=0$ if

$$
\text { (a) } \mu_{r} H_{j k}^{r}=\mu \mu_{j k} \quad \text { (b) } \mu_{j}=\psi \lambda_{j} \text {, }
$$

where $\psi$ is a scalar. Also $£ \mathrm{H}_{j k}^{i}=0$ if and only if $£ \mathrm{H}_{j \mathrm{kh}}^{i}=0$.

This leads to

Theorem 4.3. A special recurrent transformation of the second kind in an NPR-Finsler space is a curvature collineation if (4.30) holds.

In view of (4.21), we have

$$
£ G_{j k}^{i}=\left(\mathfrak{B}_{j} \mu_{k}+\mu_{j} \mu_{k}+\mu \dot{\partial}_{j} \mu_{k}\right) v^{i}+H_{m j k}^{i} v^{m}
$$

which gives

$$
£ G_{j k}^{i}=\left(\mathfrak{B}_{j} \mu_{k}+\mu_{j} \mu_{k}+\mu \dot{\partial}_{j} \mu_{k}\right) v^{i}+\lambda_{j} X_{k}^{i}
$$

where $X_{k}^{i}=\dot{\partial}_{k} X^{i}$.

This shows that a special recurrent transformation of the second kind is an affine motion if

$$
\left(\mathfrak{B}_{j} \mu_{k}+\mu_{j} \mu_{k}+\mu \dot{\partial}_{j} \mu_{k}\right) v^{i}=-\lambda_{j} X_{k}^{i} .
$$

Transvecting this equation by $\dot{x}^{k}$, we get

$$
\left(\mathfrak{B}_{j} \mu_{k}+\mu_{j} \mu_{k}\right) \dot{\chi}^{k} v^{i}=-\lambda_{j} X^{i} .
$$

Transvecting this equation by $y_{i}$, we have

$$
\left(\mathfrak{B}_{j} \mu_{k}+\mu_{j} \mu_{k}\right) \dot{x}^{k}=0
$$

for $y_{i} v^{i} \neq 0$ and $y_{i} x^{i}=0$.

Using (4.35) in (4.34), we get $X^{i}=0$. Therefore $X_{k}^{i}=0$.

Using $X_{k}^{i}=0$ in equation (4.33), we get

$$
\mathfrak{B}_{j} \mu_{k}+\mu_{j} \mu_{k}+\mu \dot{\partial}_{j} \mu_{k}=0 .
$$

Thus (4.33) implies (4.36). Conversely if (4.36) holds, its skew symmetric part gives

$$
\mu_{j k}=\mathfrak{B}_{j} \mu_{k}-\mathfrak{B}_{k} \mu_{j}=0 .
$$

Using this in (3.2) we get $\mathrm{H}_{j \mathrm{kh}}^{i} \nu^{h}=0$, which implies $\mathrm{H}_{\mathrm{m} j \mathrm{k}}^{\mathrm{i}} \nu^{\mathrm{m}}=0$.

Therefore $X_{k}^{i}=0$.

Hence we conclude:

Theorem 4.4. A special recurrent transformation of the second kind in an NPR-Finsler space is an affine motion if $\mathfrak{B}_{j} \mu_{k}+\mu_{j} \mu_{k}+\mu \dot{\partial}_{j} \mu_{k}=0$.

$$
\text { Received: September 2010. Revised: May } 2011 .
$$




\section{References}

[1] Pandey, P. N., A recurrent Finsler manifold admitting special transformations, Progress of Mathematics, 13 (1979), 85-98.

[2] Pandey, P. N., On NPR-Finsler manifold, Ann. Fac. Kinshasha, 6 (1980), 65-77.

[3] Pandey, P. N., Affine motion in a recurrent Finsler manifold, Ann. Fac. Kinshasha, 6 (1980), 51-63.

[4] Pandey, P. N., Some identities in an NPR-Finsler manifold, Proc. Nat. Acad. Sci. (India), 51 (1981), 105-109.

[5] Pandey, P. N., Certain types of affine motion in a Finsler manifold, Colloquium Mathematicum, 49 (1985), 243-252.

[6] Rund, H., The Differential Geometry of Finsler spaces, Springer-Verlag, Berlin, 1959.

[7] Yano, K., The theory of Lie derivatives and its applications, North Holland Publ. Co., Amsterdam, 1957. 